\title{
Variations
}

Variations

Revue internationale de théorie critique

$23 \mid 2020$

Pour une gauche érotique

\section{Sur le problème de la prédiction dans les sciences sociales (1933)}

\section{Max Horkheimer}

Traducteur : Emmanuel Chaput

\section{OpenEdition}

Journals

\section{Édition électronique}

URL : http://journals.openedition.org/variations/1543

DOI : 10.4000/variations. 1543

ISSN : 1968-3960

\section{Éditeur}

Les amis de Variations

\section{Référence électronique}

Max Horkheimer, «Sur le problème de la prédiction dans les sciences sociales (1933) », Variations [En ligne], 23 | 2020, mis en ligne le 01 septembre 2020, consulté le 07 septembre 2020. URL : http:// journals.openedition.org/variations/1543; DOI : https://doi.org/10.4000/variations.1543

Ce document a été généré automatiquement le 7 septembre 2020.

Les ami•e•s de Variations 


\title{
Sur le problème de la prédiction dans les sciences sociales (1933) ${ }^{1}$
}

\author{
Max Horkheimer \\ Traduction : Emmanuel Chaput
}

1 Que la question de la prévision (Voraussicht) ${ }^{2}$ sociologique soit soumise à ce débat est déjà en cela une bonne idée, puisqu'il se révèle clairement par cela que la sociologie participe elle aussi à la crise culturelle générale. La capacité (Möglichkeit) de prévision est bien pour toute science la pierre de touche du vrai (Wirklichen). L'avis selon lequel, dans le cadre de la situation historique présente, de si grandes énergies comme celles consacrées à la sociologie, bénéficieraient à une entreprise qui ne pourrait fondamentalement qu'aider à classifier rationnellement le passé, mais qui ne pourrait pas aider à former l'avenir, [un tel avis] devrait nécessairement équivaloir à un très charmant jugement sur l'ensemble des efforts scientifiques.

2 Si ma vigilance ne m'a pas laissé tomber, la possibilité même de [faire des] pronostics n'a pas été remise en question dans les thèses présentées. Et même, toute une série de participants à ce congrès a désigné des phénomènes concrets, par rapport auxquels, à leur avis, des prédictions (Voraussagen) avec un assez haut niveau de vraisemblance pourraient être faites. Néanmoins, il me semble que l'avis dominant est davantage caractérisé par une retenue sceptique que par cette confiance en soi qui a animé la science moderne à ses débuts. Et même, dans la plupart des réponses positives, on mettra plus de poids sur les restrictions quant à la portée et le niveau de certitude de la prévision $^{3}$ que sur sa fiabilité.

3 Cette prudence se comprend aisément à la lumière de l'expérience que la sociologie a fait (ainsi que l'économie) au cours des dernières décennies et particulièrement des dernières années : dans plusieurs cas, où les ensembles systématiques de catégories des systèmes modernes établis avec sagacité portaient à l'occasion de façon immédiate sur la réalité en train de se développer, il s'est avéré que les sociologues et les économistes avaient sur ce point à peine une longueur d'avance sur la conscience générale. Souvent, c'est même l'inverse qui s'est produit: des groupes d'individus qui établissaient leurs avis sur un tout autre fondement que ce sur quoi repose la sociologie et l'économie 
dominantes de nos jours et même la plupart du temps en opposition brutale avec elles, se sont vus justifiés dans leurs jugements, alors même que les experts échouaient. [408] Il n'est dès lors pas surprenant qu'aujourd'hui chez plusieurs d'entre vous, le penchant demeure de restreindre plutôt prudemment les possibilités d'affirmation sur l'avenir et de se prononcer pour des théories bien définies.

4 En revanche, j'aimerais soutenir ici l'idée qu'aujourd'hui encore le but de la science est la connaissance des processus auxquels appartient nécessairement la dimension de l'avenir. Compte tenu justement de la tendance évoquée il ne semble pas être tout à fait inutile de souligner clairement ce qu'il y a de positif vis-à-vis du doute sceptique. Il se révèle par cette occasion que la prévision ne forme pas une exception par rapport à la plupart des catégories logiques ou théorético-scientifiques: son caractère, le sens de son application, ses possibilités, le degré de vraisemblance atteint ne dépendent pas simplement du bon sens et de la compétence des sociologues, mais aussi et même plutôt de la structure des rapports sociaux de son temps. Un traitement anhistorique du problème de la prédiction (Vorhersage) présuppose un rapport statique entre la science et son objet, dans le cas présent entre la théorie sociologique et les processus sociaux. Une telle posture est cependant depuis longtemps surmontée par la philosophie contemporaine. La philosophie a même démasqué et rejeté la doctrine générale de la contradiction anhistorique entre sujet et objet, à tel point que ces deux pôles de l'acte de connaissance dans leurs rapports dynamiques sont eux-mêmes inclus dans au sein du processus historique. La détermination à chaque fois possible de l'avenir qui, je le répète, appartient à part entière aux velléités d'une théorie scientifique de la société, dépend par conséquent du développement des rapports propres à l'ensemble de la société.

5 Pour en discuter en peu de mots, je partirai de la distinction qu'a faite le secrétaire de ce congrès, le professeur Duprat ${ }^{4}$ dans son «Introduction à l'étude de la prévision sociologique $»^{5}$ : la distinction entre "prévision» et «prédiction $»^{6}$. La science de la nature connaît les deux types de jugements, tant la prévision qui se rapporte à des " modèles (Typen) abstraits » que la prédiction qui se rapporte à des « actes concrets ou des événements ». Une théorie qui voudrait affirmer qu'il serait possible de mener la science moderne qu'au niveau de prévisions, mais non au niveau de prédictions entendues en ce sens - et c'est ainsi qu'on pourrait facilement comprendre l'article mentionné - commettrait dans les faits une erreur. Ce sont justement les prédictions, que visent en fin de compte les sciences de la nature comme toute science en général. Les « modèles abstraits » au sens de la prévision sont des lois et ont en tant que telles en substance constamment une forme conditionnelle. Elles veulent dire que toujours lorsque certaines conditions déterminées sont données dans la réalité, des événements déterminés doivent nécessairement se produire. C'est ainsi par exemple une prévision de la science de la nature que l'or se dissout constamment lorsqu'on le plonge dans l'eau régale, mais pas lorsque par exemple on le plonge dans l'acide sulfurique dilué. D'autres prévisions portent sur le fait que dans certaines sortes de fer connues, des modifications dans la forme se produisent lorsqu'un effet de force d'une grandeur déterminée [409] est exercé. Ces énoncés, certains et apodictiques, sont certainement de pures prévisions, car elles ne disent absolument rien à propos de quand et si même les conditions exigées pour cela ne seront jamais données. Sur ce point, j'en conviens avec monsieur Duprat. 
6 Mais je ne suis pas à même de conclure à ce sujet que par conséquent des prédictions pour l'avenir, comme celles qu'a tentées Marx par exemple, devraient être à peu près impossibles, en tout cas pour les sciences molles. Les lois ne sont pas en effet le but de l'activité scientifique, mais bien un simple outil de travail. Au final, l'essentiel est toujours de passer des formules sous forme de lois abstraites à des jugements existentiels et concrets. Et ceux-ci n'impliquent simplement, pour l'ensemble du domaine des sciences naturelles, que des affirmations sur le passé ou le présent, mais toujours en même temps également des prédictions pour le futur. C'est ainsi que les propositions hypothétiques des exemples mentionnés emportent pour la première fois leur signification réelle, lorsqu'on déclare dans un cas précis d'une substance donnée : "Ceci est de l'or». Cette déclaration englobe donc nécessairement, lorsque que la loi hypothétique mentionnée est absolument connue, l'affirmation que ce morceau de métal présent ne se dissout effectivement pas dans de l'acide citrique dilué, mais [se dissout] complètement dans de l'eau régale. L'expérimentateur dans l'auditoire fait ainsi la prédiction: «Je dépose premièrement la pépite dorée dans cet acide et elle ne va pas se dissoudre; je la dépose ensuite dans cet autre acide, et elle va se dissoudre ». De même, la proposition générale évoquée précédemment à propos de la transformation dans la forme du fer par l'introduction d'un effet de force déterminé constitue aussi la présupposition d'une prédiction. Elle s'exprime par exemple dans le calme du chauffeur de locomotive qui laisse mugir le moteur de son train sur un nouveau pont et sait : « il ne va pas briser, car il se compose d'une certaine sorte de fer et que celui-ci supporterait même une charge bien plus grande ». Comme nous l'avons dit, la science de la nature et même la science en sa totalité impliquent de tels jugements existentiaux. Elles incluent constamment des affirmations [portant] sur l'ensemble des dimensions temporelles. Avec une simple remarque sur une des choses au sein de la nature, une prédiction est en même temps posée : «Ceci est une craie » veut dire qu'elle va produire des traits sur le tableau noir. "Ceci est une cerise» veut dire que tu pourras la manger. "Le thermomètre chute en deçà de $0^{\circ} \mathrm{C}$ " signifie en même temps que l'eau gèlera. Le présent, le passé et l'avenir de l'objet jugé sont dans chaque phrase impliqués conjointement, notamment parce que les segments de la perception ne peuvent nécessairement pas coïncider avec la structure temporelle de l'événement perçu. Bien sûr, nous perdons par le passage de la formule de loi abstraite aux propositions concrètes sur les choses réelles, le savoir absolu. Il est possible que ce morceau de métal doré n'ait absolument pas été de l'or, le pont peut s'effondrer, puisque la matière ferreuse n'est pas irréprochable, la cerise peut avoir été une belladone et même l'eau pourrait éventuellement, à la suite de transformations atmosphériques, préserver son état liquide en deçà de $0^{\circ} \mathrm{C}$. Cela est certainement tout à fait vrai, mais je voulais simplement faire remarquer ici que le sens des propositions abstraites doit s'accomplir dans des propositions concrètes, que le sens de toute prévision doit s'accomplir en des prédictions. Si la signification des abstractions [410] n'est pas elle-même contrôlée par le biais de sa perpétuelle application pratique et modifiée par les circonstances, elles doivent nécessairement devenir étrangères ${ }^{7}$ à la réalité et en fin de compte devenir non seulement inutiles, mais même fausses.

7 L'application pour la sociologie se révèle aisément. La proposition selon laquelle, dans le cadre d'une économie de libre-marché, des crises doivent nécessairement naître et que tout aussi nécessairement des monopoles doivent émerger qui accentueront encore davantage ces crises, est une prévision. L'avis selon lequel ces conditions sont présentement données, à savoir que nous vivons dans un tel type d'économie, implique 
déjà la prédiction que les crises ne connaîtront pas d'atténuation durable, même par intermittence. Elles établissent un pronostic historique sur l'autodépassement (Selbstaufhebung) de l'économie libérale et l'accentuation des contradictions sociales. Cette théorie en elle-même ne fait pas ici l'objet de la discussion, j'ai simplement voulu montrer par le biais de cette remarque, comment ces deux types de jugements, la prévision et la prédiction, vont nécessairement de pair également au sein de la sociologie. La prévision hypothétique, à savoir donc la théorie, dans ce cas-ci la doctrine du rapport entre le type d'économie et les crises, dépend quant à son sens et son degré de vérité de sa réalisation historique, tout comme elle détermine elle-même, à l'inverse, nos perceptions, nos jugements existentiaux concrets et même nos actes pratiques.

J'attends ainsi de la sociologie, véritablement toute une série d'objections par rapport à l'application de ce raisonnement méthodologique. Je vais simplement en dégager une seule afin d'y répondre : la capacité de prédiction de mes exemples tirés des sciences de la nature ne provient-elle pas de ce simple fait que, tout simplement, celui qui pose l'énoncé est dans la position de provoquer la validité de la loi même? C'est seulement dans la mesure où ce chimiste se décide à jeter effectivement l'or dans l'eau régale qu'il peut prédire qu'il sera en effet dissous. C'est seulement dans la mesure où je veux effectivement écrire avec la craie que ma prédiction à propos des traits sur le tableau est valide. En d'autres mots, dans la nature, la prédiction se rapporte à l'expérimentation arbitraire et justement parce qu'il n'y aurait pas d'expérimentation chez elle, la sociologie devrait s'abstenir de tels énoncés. Maintenant, je crois que vous avez déjà remarqué que l'objection concerne seulement certains cas particuliers et non le principe. Le chauffeur de locomotive ne réalise aucune expérimentation avec sa machine à pleine vitesse, puisque les forces de la nature ne sont pas à ce point en son pouvoir qu'il puisse forcer le train à demeurer sur le pont et ainsi être en mesure d'annoncer: "il ne brisera pas ». Et l'eau gèle dans la nature à un certain degré déterminé sans qu'on n'y soit absolument pour rien. Non, il y a de vastes domaines du savoir où nous ne pouvons absolument rien dire : « dans le cas où ces conditions sont données, en découle de ce fait également cet événement attendu, sans que notre volonté même fût en jeu ». D'un point de vue purement logique cette objection est ainsi négligeable.

Pour la sociologie, cela entre néanmoins en ligne de compte. Certes, il est inexact de dire que la prédiction ne serait possible que si l'apparition des [411] conditions nécessaires dépend de celui-là même qui prédit, mais la prédiction sera d'autant plus vraisemblable qu'elle dépend d'un rapport nécessaire avec la volonté de l'être humain, c'est-à-dire d'autant plus que l'effet prédit n'est pas un produit de la nature aveugle, mais bien l'effet de décisions rationnelles. Parce que la sociologie a à voir avec les processus sociaux, nous pourrions ainsi croire que ses prédictions devraient être aussi pertinentes que celles de n'importe quelle autre science, puisque la société se compose elle-même justement d'êtres humains agissants. À partir de réflexions similaires, Giambattista Vico a aussi interprété, contre Descartes et son école, l'histoire comme la véritable science. Si nous avons, depuis, fait l'expérience que les prédictions se laissent plus difficilement faire eu égard à la société actuelle que par rapport à la nature nonhumaine (aussermenschliche), cela ne prouve pas pour autant que le principe de Vico soit faux. Ces prédictions sont bien davantage imparfaites du fait que les processus sociaux ne sont encore en aucun cas le résultat de la liberté humaine, mais bien au contraire les conséquences naturelles de l'activité aveugle de forces antagonistes. La manière que notre société maintient et renouvelle sa vie ressemble bien plus au déroulement d'un 
mécanisme naturel qu'à une action orientée par des fins. Le sociologue se tient en cela face à cette manière comme face à un événement essentiellement étranger. Il est concerné par lui, et y participe d'une certaine manière, son devoir consiste cependant à l'accepter en tant qu'observateur, à le colliger et le décrire et si possible l'expliquer. Les processus sociaux se produisent bien sûr par la médiation de personnes, mais ils sont malgré tout vécus comme des événements détachés et fatidiques. De bonnes ou de mauvaises conjonctures, la guerre, la paix, les révolutions, les périodes de stabilité apparaissent aux êtres humains comme autant d'événements naturels que le bon ou le mauvais temps, les tremblements de terre et les épidémies. On doit chercher à les expliquer, et pourtant vos prédictions passent à bon droit comme une expression risquée.

10 Cette situation n'est appropriée ni de toute éternité, ni même pour le niveau de développement actuel des forces humaines. Les signes les plus variés se manifestent présentement pour soumettre les processus sociaux à la planification humaine. Peutêtre considérera-t-on même un jour cette époque comme la transition d'un fonctionnement purement naturel, et par-là mauvais, de l'appareil social à une œuvre commune et consciente des forces sociales. Dans tous les cas, vous serez d'accord avec moi que le manque de dépendance des événements sociaux par rapport à une volonté homogène ne doit pas être irrévocable, mais est au contraire fondé sur les propriétés structurelles spécifiques de la situation sociale actuelle. Certes, la loi - eu égard à notre problème - se formule de telle sorte qu'avec la transformation croissante de cette structure en termes d'organisation unifiée et de planification, les prédictions gagneront également un plus grand degré de certitude. Plus la vie sociale perd l'aspect d'un événement naturel aveugle et que la société atteint [un niveau] d'institutions [lui permettant] de se constituer comme un sujet rationnel, plus les processus sociaux sont également [aisés] à prédire avec déterminité. L'incertitude [412] actuelle dans les jugements sociologiques à propos de l'avenir n'est que le reflet de l'incertitude sociale actuelle en général.

11 La capacité de prédiction ne dépend pas par conséquent exclusivement de l'affinement de la méthode et de la sagacité du sociologue, mais tout autant du développement de son objet: de la transformation structurelle de la société même. Loin du fait que la prédiction serait nécessairement plus facilement possible dans le domaine de la nature non-humaine qu'au niveau de la société, elle est au contraire d'autant plus facile que son objet est moins soumis à la pure nature et davantage soumis à [l'emprise de] la liberté humaine. Car la véritable liberté humaine n'est à confondre ni avec l'inconditionnel, ni avec l'arbitraire pur, mais elle est au contraire identique à la maitrise (Beherrschung) de la nature en et hors de soi par le biais d'une décision rationnelle. Mener au fait que cette situation devienne caractéristique de la société est non seulement le devoir du sociologue, mais encore celui des forces de l'humanité allant de l'avant (vorwärtsstrebenden). Et ainsi, les efforts (Bemühen) du sociologue pour parvenir à une prédiction plus exacte transbordent du côté de l'effort (Streben) politique visant la réalisation d'une société rationnelle.

\section{Le problème de la prédiction en sociologie ${ }^{8}$}

12 Chaque science comprend des jugements ayant trait à l'avenir, non seulement par la formation de «types abstraits » et de lois (ce qu'on a appelé la "prévision »), mais par 
des "prédictions » concrètes, impliquées dans chaque loi scientifique. Les jugements hypothétiques et catégoriques (prévisions et prédictions) exercent, dans le développement des sciences, une influence mutuelle les uns sur les autres. À l'encontre de la conception traditionnelle, $\mathrm{H}$. est d'avis que la prévision scientifique est facilitée si elle porte plutôt sur des objets pour lesquels la volonté joue un rôle que si elle porte sur la nature hors de l'homme. La prédiction sociologique sera d'autant plus exacte que les événements historiques perdront le caractère de faits provenant d'un mécanisme aveugle et seront en réalité l'œuvre de la liberté de l'homme.

\section{NOTES}

1. Reproduction augmentée d'une contribution à la discussion lors $11^{\mathrm{e}}$ congrès international des sociologues à Genève, en octobre 1933 [paru dans la Zeitschrift für Sozialforschung, vol. 2, 1933, p. 407-12 (N.d.T)].

2. «Prévision » est inscrit en français entre parenthèses dans l'original (N.d.T.).

3. Les termes prévision(s) et prédiction(s) en italique tout au long du texte sont en français dans l'original (N.d.T.).

4. Guillaume-Léonce Duprat (1872-956), psycho-sociologue français inspiré tant par Durkheim que par le solidarisme. Membre actif de l'Institut international de sociologie qui assurait la publication de la Revue internationale de sociologie (N.d.T.).

5. Revue internationale de Sociologie, 1932, Nr. III-IV.

6. En français dans l'original. Horkheimer donne ensuite sa traduction allemande de ces termes : «Voraussicht » pour prévision et « Voraussage » pour prédiction (N.d.T.).

7. Horkheimer utilise ici le terme «entfremdet » qui dérive du verbe «entfremden » (aliéner) (N.d.T.).

8. Ce résumé apparaît en français et en anglais à la fin de l'original (N.d.T.).

\section{AUTEURS}

\section{MAX HORKHEIMER}

Max Horkheimer (1895-1973), philosophe et sociologue allemand. Penseur de la Théorie critique, il a dirigé l'Institut de recherches sociales (Institut für Sozialforschung) de Francfort entre 1930 et 1969. 